\title{
Utilidad del índice de resistencia renal en la valoración y evolución de la uropatía obstructiva. Estudio experimental
}

\author{
Soria Gálvez F*, Delgado Márquez MI*, Rioja Sanz LA**, Blas Marín M**, Durán Flores Mª́E***, \\ Usón Gargallo J*. \\ *Unidad de Endoscopia del Centro de Cirugía de Mínima Invasión de Cáceres. **Servicio de Urología del \\ Hospital Universitario Miguel Servet. Zaragoza. ${ }^{* * *}$ Cátedra de Anatomía Patológica de la Facultad de \\ Veterinaria de la Universidad de Extremadura.
}

Actas Urol Esp. 2007;31(1):38-42

\begin{abstract}
RESUMEN
UTILIDAD DEL ÍNDICE DE RESISTENCIA RENAL EN LA VALORACIÓN Y EVOLUCIÓN DE LA UROPATÍA OBSTRUCTIVA. ESTUDIO EXPERIMENTAL

Objetivo: El planteamiento del estudio va enfocado a determinar la efectividad y fiabilidad de la medición del índice de resistencia renal (IR) para el diagnóstico y evolución tras el tratamiento de la uropatía obstructiva. Para ello realizamos un estudio experimental con un grupo homogéneo y con un grado de obstrucción uniforme.

Material y métodos: Se emplean 15 animales de la especie porcina, todas hembras sanas. El planteamiento experimental está vertebrado en tres fases; la primera consiste en el estudio previo a la obstrucción de la unión pieloureteral unilateral mediante ureteropielografia retrógrada, nefrosonografia en modo B y dúplex-Doppler (ambos riñones) al nivel de las arterias arciformes. Posteriormente se procede a la creación del modelo animal de obstrucción. La Fase II comienza con el diagnóstico de la lesión 6 semanas después de la anterior fase, mediante los medios diagnósticos citados. Finalmente se completa el tratamiento endourológico. El seguimiento de los animales (Fase III) se realiza transcurridas 15 semanas tras la endopielotomía, valorando al igual que durante el estudio, la vía urinaria (fluoroscopia) y la afectación en ambos riñones mediante la determinación del índice de resistencia renal y su ratio.

Resultados: Todos los animales del estudio mostraron signos radiológicos y nefrosonográficos de obstrucción urinaria tras 6 semanas de la ligadura ureteral. Tras el tratamiento y seguimiento todos los animales mostraron signos de recuperación de la uropatía obstructiva. Los valores del $\Delta \mathrm{IR}$ a lo largo de las 3 fases se detallan a continuación. Fase I $\Delta \mathrm{IR}=0.01$, Fase II $\Delta \mathrm{IR}=0.11$, Fase III $\Delta \mathrm{IR}=0.02$.

Conclusiones: La valoración del IR mediante dúplex-Doppler es efectiva para la diferenciación de la dilatación obstructiva mediante técnicas no invasivas. Pero este parámetro aporta unas diferencias muy leves y además puede verse afectado por demasiados factores de modo directo o indirecto (observador, edad del paciente, anestesia, parámetros hemodinámicos, etc.), como para relegar los métodos de diagnóstico clásicos.
\end{abstract}

Palabras clave: Índice de resistencia renal. Uropatía obstructiva. Endopielotomía. Experimental. Doppler.

\section{ABSTRACT}

USEFULNESS OF RENAL RESISTIVE INDEX IN THE DIAGNOSIS AND EVOLUTION OF THE OBSTRUCTIVE UROPATHY. EXPERIMENTAL STUDY.

Objective: The study was focused on determining the effectiveness and reliability of using the renal resistance index as a method of diagnosing and monitoring the evolution of obstructive uropathy treatment. For this purpose, we conducted an experimental study on a homogenous group of animals that all had the same level of obstruction.

Material and methods: 15 healthy female pigs were used. The experiment was divided into three phases: phase I consisted of a study prior to unilateral pyeloureteral junction obstruction, performing retrograde ureteropyelography, renal Bmode ultrasound and duplex-Doppler (of both kidneys) at a level of the arcuate arteries. Then, the obstruction was performed on the animals. Phase II commenced by diagnosing the lesion, 6 weeks after the previous phase, by means of the aforementioned diagnostic methods. Finally, the endourological treatment was completed. Animals were monitored (Phase III) 15 weeks after the endopyelotomy, using the same methods as in the study, by assessing the urinary tract (fluoroscopy) and both kidneys by determining the renal resistance index and ratio.

Results: All the animals in the study showed signs of urinary obstruction on radiology and renal ultrasound 6 weeks after ureteral ligature. After treatment and follow-up, all animals showed signs of having recovered from the obstructive uropathy. Values of $\Delta$ RI during the 3 phases are detailed below. Fase I $\Delta \mathrm{IR}=0.01$, Fase II $\Delta \mathrm{IR}=0.11$, Fase III $\Delta \mathrm{IR}=0.02$.

Conclusion: RI determination using duplex-Doppler is effective for distinguishing obstructive dilatation using non-invasive techniques. However, this parameter provides very slight differences and it can also be influenced by too many direct and indirect factors (observational, patient age, anaesthesia, haemodynamic parameters, etc.), to supplant the classic diagnostic methods.

Keywords: Renal resistive index. Obstructive uropathy. Endopyelotomy. Experimental. Doppler. 
$\mathrm{E}^{\mathrm{a}}$ indice de resistencia renal (IR) es un parámetro fisiológico que indirectamente refleja el grado de resistencia en los vasos intrarrenales ${ }^{1}$. Por lo tanto es potencialmente útil para detectar enfermedades renales asociadas al incremento o disminución de la resistencia vascular renal. Platt en 1989 plantea la hipótesis de que un incremento en la resistencia vascular en riñones obstruidos podría verse reflejado en las ondas del Doppler, produciendo una bajada en el flujo diastólico y un incremento en el índice de resisten$\mathrm{cia}^{2}$. Esto es de gran utilidad para la diferenciación entre dilatación no obstructiva del sistema colector renal de la dilatación debida a obstrucción ureteral, ya que esta distinción es difícil de llevar a cabo sin métodos invasivos ni radiológicos.

De su experiencia en modelos animales y humanos concluyen que el IR límite medido en arterias arcuatas o interlobulares para diagnosticar dilatación obstructiva está marcada por un IR de 0,70 , por encima de este valor la dilatación es obstructiva, con una sensibilidad del $93 \%$ y especificidad del $100 \%{ }^{2}$.

Sin embargo después de los primeros resultados prometedores, las recientes investigaciones no han corroborado estos hallazgos positivos, posiblemente debido a que estos trabajos son poco homogéneos ${ }^{3}$. Por lo que nuestro objetivo es valorar esta técnica diagnóstica en un modelo porcino para determinar su fiabilidad y de este modo disponer de un test no invasivo capaz de identificar un incremento en la resistencia vascular intrarrenal para diferenciar pacientes con hidronefrosis sin significación fisiológica de pacientes con uropatía obstructiva verdadera.

\section{MATERIAL Y MÉTODOS}

Se seleccionan 15 hembras sanas de la especie porcina con pesos comprendidos entre 35 y 40 $\mathrm{Kg}$. El empleo de esta especie es debido a la gran similitud demostrada entre el tracto urinario superior de esta especie con la especie humana ${ }^{4}$. Todos los procedimientos se realizan bajo el mismo protocolo anestésico, mediante anestesia general inhalatoria (Isofluorano) y control analgésico en su recuperación postoperatoria (Ketorolaco). El estudio se efectúa tras la aprobación por parte del Comité de ética de experimentación animal del
Centro de Cirugía de Mínima Invasión de Cáceres, cumpliendo la normativa europea sobre protección de los animales empleados para experimentación y otros fines científicos. Tras el periodo preceptivo de adaptación y cuarentena de los animales se inicia el estudio.

Diseñamos el estudio en tres fases:

\section{Fase I}

El estudio comienza con la valoración del tracto urinario superior mediante nefrosonografia, determinando también mediante dúplex-Doppler sonográfico el índice de resistencia renal a nivel de las arterias arciformes de la unión corticomedular en ambos riñones. Estos sistemas dúplex permiten obtener simultáneamente una imagen morfológica bidimensional y un registro Doppler del vaso estudiado. Tras corregir el ángulo de incidencia con respecto al flujo sanguíneo, un mínimo de 3 ondas Doppler consecutivas e iguales son obtenidas para llevar a cabo la determinación del IR. Todos los animales son valorados mediante la escala de velocidad más pequeña posible para provocar las ondas más largas y evitar errores que puedan ser causados por ondas pequeñas. Los valores del IR de cada riñón en cada individuo son posteriormente relacionados mediante el $\Delta \mathrm{IR}=\mathrm{IR}$ riñón dilatado menos IR riñón no dilatado.

Consecutivamente completamos una cistografía de compresión para descartar reflujo vesicoureteral en los animales, si este se produce los animales son desechados del estudio. A continuación se realiza una urografía excretora y una ureteropielografia retrógrada valorando el trayecto de toda la vía excretora superior.

Finalmente y en la misma fase realizamos un abordaje laparoscópico para completar el modelo de obstrucción urinaria descrito por Nakada et al. cambiando el tipo de sutura empleado por ác. poliglicólico ${ }^{5}$.

\section{Fase II}

Transcurridas 6 semanas procedemos al diagnóstico y tratamiento de la obstrucción. Completando los mismos estudios de imagen que en la fase anterior.

Todos los animales sufrieron idéntico tratamiento endourológico mediante endopielotomía 
retrógrada con dispositivo Acucise ${ }^{\circledR}$. Disponiendo posteriormente un catéter ureteral doble pigtail de $7 \mathrm{~F}$, que es retirado vía cistoscópica a las 3 semanas.

\section{Fase III}

Quince semanas tras el tratamiento endourológico se procede a efectuar el estudio de seguimiento, valorando los mismos parámetros hemodinámicos y fluoroscópicos que en las anteriores fases.

\section{Análisis estadístico}

Los resultados experimentales son expresados como la media $\pm \mathrm{DV}$ (desviación estándar). Se sometieron a análisis estadístico mediante ANOVA los resultados del IR y de $\Delta$ IR comparando las tres fases entre ellas. En el caso de encontrar diferencias estadísticamente significativas entre las medias de las distintas variables, las diferencias entre medias se establecieron "a posteriori" mediante una prueba de Tukey a un nivel de significación $\mathrm{p}<.05$ (SPSS 10.0 para Windows, SPSS Inc, Chicago, EE.UU).

\section{RESULTADOS}

Todos los animales del estudio mostraron antes del modelo de uropatía obstructiva ausencia de lesiones tras la valoración sonográfica y fluoroscópica. Las curvas de Doppler muestran baja resistencia, ausencia de turbulencias y un pico sistólico corto y una diástole alargada. El IR en el riñón de estudio muestra una media de $0,52 \pm 0,08$ y en el riñón contralateral $0,51 \pm 0,07$, con un $\Delta \mathrm{IR}=0,01$, (Fig. 1).

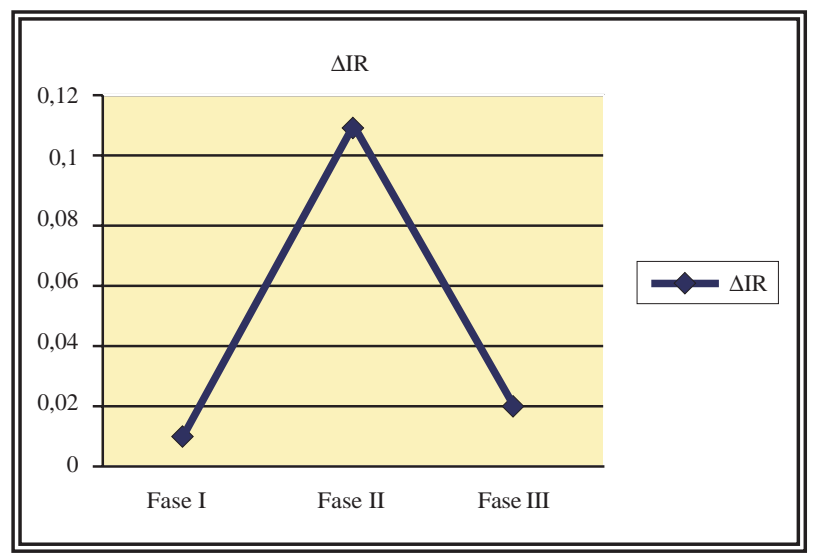

FIGURA 1
Transcurrido el tiempo necesario para la degradación de la sutura (Fase II), la valoración fluoroscópica y nefrosonográfica determina la aparición de uropatía obstructiva del tracto superior en todos los animales del estudio. El IR en el riñón de estudio muestra una media de $0,57 \pm 0,51$ y en el riñón contralateral $0,46 \pm 0,64$, con un $\Delta \mathrm{IR}=0,11$. Un único animal del estudio presenta un riñón lesionado con un IR menor a la media de la Fase I, concretamente 0,51. A diferencia de lo evidenciado en la fase anterior se aprecia en el riñón lesionado un acortamiento de la curva que refleja la diástole en el Doppler.

Tras el seguimiento final (Fase III) 14 de los 15 animales del estudio muestran una recuperación de la uropatía. La valoración del IR en el riñón que sufre el tratamiento es $0,46 \pm 0,07$ y $0,44 \pm 0,05$ en el riñón contralateral, con un $\Delta \mathrm{IR}=0,02$ (Fig. 1). Únicamente en un riñón del estudio en esta fase el IR supera la media de la fase II, coincidiendo exactamente con el animal que no muestra recuperación (IR=0,61).

Tras el estudio estadístico encontramos diferencias estadísticamente significativas entre el IR del riñón lesionado en fase II frente a su contralateral en la misma fase, frente al propio riñón lesionado en la fase III y también frente al riñón contralateral en la fase III. Tras la valoración estadística del $\Delta \mathrm{IR}$ encontramos diferencias estadísticamente significativas entre la fase I y la II $(\mathrm{p}=0,003)$ y entre la II y la III $(\mathrm{p}=0,012)$.

\section{DISCUSIÓN}

La dilatación del tracto urinario superior tiene actualmente una definición y manejo que está sujeta a creciente debate y su significación clínica es a menudo cuestionada ${ }^{6}$.

Los cambios hemodinámicos renales que encontramos en este estudio tienen su base fisiopatológica como consecuencia de que en el inicio de una obstrucción completa se produce un incremento transitorio en el flujo sanguíneo renal y vasodilatación mediada por prostaglandinas, no estando asociado a incrementos en IR. Posteriormente se produce un incremento en la resistencia vascular renal, disminución del flujo renal y elevación en la presión ureteral, coincidiendo con una elevación del IR. Finaliza con una fase de disminución de la presión ureteral y una marcada 
elevación de la resistencia vascular renal, provocada por gran cantidad de factores vasoactivos $\mathrm{u}$ hormonas, como son los factores tromboxano $\mathrm{A}_{2}$ $\left(\mathrm{TXA}_{2}\right)$ y la angiotensina II, producidos en condiciones de obstrucción por un infiltrado de macrófagos, con una marcada vasoconstricción intrarrenal, elevada resistencia vascular renal y disminución del flujo renal sanguíneo. Estos cambios no se observan en los riñones contralaterales sanos en modelos animales de obstrucción. Debido a esta vasoconstricción se provoca un incremento en la resistencia vascular causante de una marcada reducción del flujo diastólico comparado con el componente sistólico, que incrementa el $\mathrm{IR}^{7,8}$. Estos datos de obstrucción renal son los esperados para su detección mediante examen dúplex-Doppler.

Es importante resaltar que nuestros resultados coinciden con el trabajo de Pope et al que describe un índice de resistencia basal en la especie porcina de $0,53 \pm 0,10^{6}$. Ya que en este aspecto existen grandes discrepancias que dificultan la comparación entre estudios experimentales debido a la diversidad de valores basales como $0,63 \pm 0,09$ y $0,58 \pm 0,05^{1,9}$. Esto es debido a que el IR como mencionamos anteriormente se ve afectado por muchos factores al que también se puede añadir la dependencia del observador ${ }^{6,9}$.

Evidenciamos tras la estimación del $\Delta \mathrm{IR}$ en la fase II un valor superior a 0,08-0,1 que según el trabajo de Kessler et al. es un signo positivo de uropatía obstructiva, y una disminución de este en la última fase del estudio coincidiendo con la ausencia de signos fluoroscópicos y ecográficos de obstrucción ${ }^{10,11}$. Esta disminución del $\Delta \mathrm{IR}$ indica que los agentes vasoactivos que provocan el incremento de la resistencia vascular renal han desaparecido por efecto de la endopielotomía.

A diferencia de Frokiaer y Ulrich que no aprecian alteraciones en el riñón contralateral sano, nosotros coincidiendo con Shokeir y et al. encontramos modificaciones en el IR en ese riñón, ya que se aprecia una disminución desde la fase II $^{12}$ 14. Este hecho se debe a una acción compensatoria que disminuye la resistencia vascular del riñón contralateral para incrementar su rendimiento, que subsanaría la disminución de la tasa de filtración glomerular que acontece en el riñón obstruido. Es conocido el hecho de que en pre- sencia de una disminución de la función renal unilateral por una patología presente o por ausencia de un riñón, el riñón sano remanente sufre una hipertrofia compensadora, estos cambios y el grado de éstos está relacionado con la cantidad de tejido lesionado en el riñón contralateral patológico ${ }^{15,16}$ La hipertrofia renal compensadora comienza inmediatamente después de establecerse la lesión del riñón contralateral.

Encontramos en nuestro estudio que existe una interrelación fisiológica entre los dos riñones, aunque sólo uno de ellos se vea afectado por la obstrucción. Este hecho nos hace inclinarnos por la valoración de ambos riñones y no por medidas puntuales del IR en el riñón lesionado. Por lo tanto consideremos que la estimación del $\Delta \mathrm{IR}$ puede reducir las limitaciones del IR en el diagnóstico de la uropatía obstructiva.

La sensibilidad de esta técnica es del $66 \%$ para nuestro estudio, estos valores son más altos que los descritos en otros trabajos $(19,3 \%$ y $42 \%)^{17,18}$, pero más bajo que el primero estudio de Platt et al. $(90 \%)^{2}$. Con esta sensibilidad y aunque encontramos diferencias estadísticamente significativas importantes consideramos que el IR se mueve en unos valores demasiado próximos por lo que su utilidad clínica es todavía limitada.

Además consideramos al igual que otros autores que el IR es un valor que puede verse alterado por demasiados factores ajenos a la obstrucción urinaria superior como son:

En el supuesto de obstrucciones agudas en las que no da tiempo a que el IR se eleve. Ya que por lo menos son necesarias 18-24 horas. En estos casos puede ser de gran utilidad la comparación entre los dos riñones del paciente ${ }^{1,10}$.

También en las obstrucciones crónicas con marcada pérdida del parénquima se ha evidenciado que no hay alteración del IR, debido posiblemente a que no existe vasoconstricción a esos niveles ${ }^{1}$.

Una importante limitación esta relacionada con la edad ya que se ha demostrado que el IR es altamente dependiente de la edad en humanos. Ya que el flujo cortical disminuido en las primeras semanas de vida es probablemente debido al gran número de glomérulos inmaduros y esto podría provocar el elevado IR en neonatos ${ }^{21}$.

Causas nefrológicas como necrosis tubular y nefritis intersticial ${ }^{1}$. 
Otros factores son marcada hipotensión, el grado de hidratación, disminución de la frecuencia cardíaca, colecciones de fluidos subcapsulares o perirrenales y neuropatía diabética ${ }^{20}$.

Reducciones en el flujo sanguíneo renal de hasta el 10\% de media en la línea basal pueden ocurrir sin cambio en el IR como se ha descrito en un modelo animal ${ }^{3,17,22}$.

Tras nuestros resultados encontramos que a diferencia de otros estudios ${ }^{6,22}$, la valoración ultrasonográfica en modo Doppler de las arterias intrarrenales es una técnica capaz de detectar los cambios hemodinámicos que acontecen durante una dilatación obstructiva y en la evolución tras el tratamiento. Pero consideramos que esta técnica no demuestra una gran sensibilidad y actualmente no puede relegar los métodos de diagnóstico clásicos, aunque si se muestra como una buena prueba complementaria en el estudio nefrosonográfico en pacientes en los que este contraindicada la exposición a radiaciones ionizantes o alérgicas a los medios de contraste.

\section{REFERENCIAS}

1. Rawashdesh YF, Djurhuus JC, Mortensen J, Horlyck A, Frokiaer J. The intrarenal resistive index as a pathophysiological marker of obstructive uropathy. J Urol. 2001;165(5): 1397-1404.

2. Platt JF, Rubin JM, Ellis JH. Distinction between obstructive and nonobstructive pyelocaliectasis with duplex Doppler sonography. Am J Roentgenol 1989;153(5):997-1000.

3. Tublin M, Dodd G, Verdile V. Acute renal colic: Diagnosis with duplex Doppler US. Radiology 1994;193(3):697-701.

4. Schwalb DM, Eshghi M, Davidian M. Morphological and physiological changes in the urinary tract associated with ureteral dilation and ureteropyeloscopy: an experimental study. J Urol. 1993;149(6):1576-1585.

5. Nakada SY, Soble JJ, Gardner SM, Wolf JS, Figenshau RS, Pearle MS, et al. Comparison of Acucise endopyelotomy and endoballoon rupture for management of secondary proximal ureteral stricture in the porcine model. J EndoUrol. 1996; 10(4):311-318.

6. Pope JC, Hernaz-Schulman M, Showalter PR, Cole TC, Schrum FF, Szurkus D, et al. The value of Doppler resistive index and peak systolic velocity in the evaluation of porcine renal obstruction. J Urol. 1996;156(2):730-733.

7. Kim WS, Han TI, Kim SH, Park M, Kim IO, Yeon KM. Renal Doppler ultrasound examination of ureteral obstruction in rabbits: effects of different sites and degrees of obstruction on renal resistive index. Invest Radiol 2004;39(9):531-536.

8. Okada T, Yoshida H, Iwai J, Matsunaga T, Yoshino K, Ohtsuka Y, et al. Pulsed Doppler sonography of the hilar renal artery:differentiation of obstructive from nonobstructive hydronephrosis in children. J Pediatr Surg 2001;36(3): 430-435.

9. Rawashdeh YF, Mortensen J, Horlyck A, Olsen KO, Fisker RV, Schroll L, et al. Resistive index: an experimental study of the normal range in the pig. J. Scand J Urol. Nephrol 2000;34(1):10-14.
10. Cole TC, Brock JW, Pope JC, Schrum FF, Milam DF, Flickinger JE, et al. Evaluation of renal index, maximum velocity, and mean arterial flow velocity in a hydronephrotic partially obstructed pig model. Invest Radiol 1997; 32(3): 154-160.

11. Kessler RM, Quevedo H, Lankau CA, Ramírez-Seijas F, Cepero-Akselrad A, Altman DH, et al. Obstructive vs nonobstructive dilatation of the renal collecting system in children:Distintion with duplex sonography. Am J Roentgenol 1993;160(2):353-357.

12. Brkljacic B, Kuzmic AC, Dmitrovic R, Rados M, Vidjak V. Doppler sonographic renal resistance index and resistance index ratio in children and adolescents with unilateral hydronephrosis. Eur Radiol 2002;12(11):2747-2751.

13. Frokiaer J, Knudsen L, Nielsen AS, Pedersen EB, Djurhuus JC. Enhanced intrarenal angiotensin II generation in response to obstruction of the pig ureter. Am J Physiol 1992;263(3):F527-F533.

14. Ulrich JC, York JP, Koff SA. The renal vascular response to acutely elevated intrapelvic pressure: resistive index measurements in experimental urinary obstruction. J Urol. 1995; 154(3): 1202-1204.

15. Shokeir AA, Nijman RJ, el-Azab M, Provoost AP. Partial ureteric obstruction:a study of Doppler ultrasonography and diuretic renography in different grades and durations of obstruction. Br J Urol. 1996;78(4):829-835.

16. Espuela R. Fisiopatología de la obstrucción del tracto urinario superior. En: Jiménez-Cruz FJ, Rioja LA. Tratado de Urol.ogía. Barcelona: J.R. Prous S.A, 1993;369-383.

17. O`Sullivan DC, Dewan PA, Guiney EJ. Compensatory hypertrophy effectively assesses the degree of impaired renal function in unilateral disease. Br J Urol. 1992;69(4): 346-350.

18. Lee HJ, Kim SH, Jeong YK. Doppler sonographic resistive index in obstructed kidneys. J Ultrasound med 1996; 15(9):613-618.

19. Older RA, Stoll HL, Omary RA, Watson LR. Clinical value of renovascular resistive index measurement in the diagnosis of acute obstructive uropathy. J Urol. 1997;157(6): 2053-2055.

20. Platt JF. Duplex doppler evaluation of native knidney dysfunction:obstructive and nonobstructive disease. Am J Roentgenol 1992;158(5):1035-1042.

21. Drudi FM, Pretagostini R, Padula S, Donnetti M, Giovagnorio F, Mendicino P, et al. Color Doppler ultrasound in renal transplant: role of resistive index versus renal cortical ratio in the evaluation of renal transplant diseases. Nephron Clin Pract 2004;98(3):67-72.

22. Murat A, Akarsu S, Ozdemir H, Yildirim H, Kalender O. Renal resistive index in healthy children. Eur $\mathrm{J}$ Radiol 2005;53(1):67-71.

\section{Agradecimientos}

A la Fundación para la Investigación en Urología (Asociación Española de Urología) y al Plan Regional de Investigación (Consejería de Infraestructuras y Desarrollo Tecnológico de la Junta de Extremadura, 2PR04A053). A A. Santos, M. Borrega, S. Bravo y B. Loscertales por su importante contribución en este estudio.

\footnotetext{
Correspondencia autor: Dr. F. Soria Gálvez. Unidad de Endoscopia. Centro de Cirugía de Minima Invasión. Campus Universitario. Avda. de la Universidad, s/n. 10071 Cáceres. Tel.: 927181032 E-mail autor: fsoria@ccmi.es Información artículo: Trabajo experimental - Uropatía obstructiva (Trabajo aceptado septiembre 2006)
} 